\author{
Brújula. Semilleros de Investigación \\ Volumen 8, Número 16, julio-diciembre, 2020. pp. 7-14 \\ Bogotá D. C., Colombia \\ ISSN 2346-0628 (en línea) \\ https://doi.org/10.21830/23460628.25
}

Seguridad y Defensa

\title{
Los libros blancos en Suramérica: un libro blanco en Colombia
}

\author{
Jorge Chacón Moreno \\ Juan Rojas Azuero \\ Sara Quintero Cordero \\ Escuela Militar de Cadetes "General José María Córdova”
}

\section{RESUMEN}

El objetivo de este artículo es analizar el Libro blanco de defensa nacional de Brasil, la forma como ha evolucionado el concepto de seguridad y cómo puede ser aplicado en Colombia. El trabajo consiste en un análisis crítico conceptual centrado en la observación de ciertas variables, como la definición de seguridad, los sectores de seguridad, qué tipo de amenazas se van a enfrentar y, especialmente, el alcance y la importancia de las Fuerzas Militares. La elaboración de un nuevo libro blanco de defensa nacional hace necesario estudiar el Estado brasileño y la región, de tal manera que sea posible visualizar los probables cambios que pueden afectar la política de defensa colombiana y sus aspectos fundamentales. De esta manera se concluye que el libro blanco de Brasil es una herramienta útil para extraer elementos de diálogo que permitan un trabajo regional frente a las nuevas amenazas en los países y sus vecinos, particularmente en Colombia.

\section{PALABRAS CLAVE}

Defensa; Fuerzas Militares; identidad; libro blanco; jugador global; Suramérica.

\section{CITACIÓN}

Quintero Cordero, S., Chacón Moreno, J., \& Rojas Azuero, J. (2020). Los libros blancos en Suramérica: un libro blanco en Colombia. Revista Brújula de Investigación, 8(16), 7-14. https://doi.org/10.21830/23460628.25 


\section{Introducción}

Durante muchos años existió un inconformismo por las políticas de defensa nacional en Colombia. En este contexto, y con el propósito de plantear un horizonte de mejora a estas políticas, en este artículo se analiza el libro blanco de defensa nacional de Brasil, un actor de gran envergadura en la región por su poder militar y económico, así como por su capacidad de influir en el sistema internacional.

No obstante, cabe anotar que la importancia geopolítica regional y mundial del "Gigante Suramericano” ha disminuido, algo que era impensable hace unos años (Grabendorff, 2010). Debido a una infrecuente combinación de desastres políticos y económicos de naturaleza estructural y coyuntural, Brasil necesitará algunos años para recuperar su papel geopolítico en la región y alcanzar el que le correspondería a nivel global por razones de su tamaño y volumen de recursos humanos y materiales (Grabendorff, 2016).

Por esta razón, Brasil implementó en el año 2012 el libro blanco de seguridad y defensa, mediante el cual buscaba renacer como un referente para la región en temas de seguridad y defensa, así como recuperar su papel como líder suramericano en el contexto geopolítico, que en el siglo XXI afronta nuevos desafíos por los constantes cambios de la vida política de los Estados.

En este sentido, analizar los elementos estratégicos e intereses nacionales disuasivos consignados en el libro blanco de seguridad y defensa de Brasil permite entender el contexto geopolítico y militar que proyecta para Suramérica en su lucha contra las distintos problemáticas que afronta el continente, como el cambio climático y el terrorismo internacional, y que planea superar proyectando una amistad regional en beneficio de los países comprometidos.
Con base en este análisis se espera abrir un debate sobre la creación de un libro blanco en Colombia para que la seguridad y defensa del Estado se encaminen a cooperar e integrarse en conceptos más regionales como defensa y cooperación internacional, en diversos ámbitos de la seguridad (humana, ciberseguridad, nuevas amenazas, etc.). Es importante resaltar que en Colombia cada cuatrienio el gobierno de turno elabora un plan de desarrollo, del cual se deriva un plan de seguridad y defensa que es dirigido por el Ministerio de Defensa de acuerdo con las políticas de seguridad del presidente. Por lo tanto, además de analizar el libro blanco de Brasil, en este artículo se comparan los planes de seguridad y defensa de los dos últimos gobiernos para tener una visión amplia y académica sobre el tema de seguridad y defensa en Colombia, así como determinar algunos lineamientos que definan con más claridad el accionar futuro de las Fuerzas Militares ahora que el país es miembro observador de la Organización del Tratado Atlántico Norte (OTAN).

Sin embargo, ¿para qué le serviría un libro blanco de defensa nacional a Colombia? Principalmente, este documento es útil para que la sociedad se ponga de acuerdo sobre qué tipo de Fuerza desea, qué características debe tener, qué misiones sería capaz de realizar y cómo debería ser la política de defensa del Estado. Además, constituye una medida de fomento de la confianza frente a la sociedad y al sector de defensa a las Fuerzas Militares.

A continuación, en la primera parte del artículo se analizan los elementos estructurales de la política de seguridad y defensa que consignó Brasil en su libro blanco. Posteriormente, se aborda la política brasileña como una herramienta útil para definir elementos de diálogo que propicien un trabajo regional para hacer frente a las nuevas amenazas en los países suramericanos, particularmente en Colombia. 


\section{Brasil y su libro blanco}

Las políticas de defensa nacional, o libros blancos de defensa, representan la construcción que hace un Estado de su defensa nacional frente a las amenazas que percibe en el contexto nacional, regional e internacional. Según Waltz (2000), en el neorrealismo los Estados siempre querrán alcanzar seguridad a través del poder y de la posición que este les otorga en la estructura del sistema internacional. Además, este autor plantea que si bien el Estado sigue siendo potencialmente el objeto de estudio, también se encuentran otras variables que explican el comportamiento de los Estados, como la interdependencia, el poder blando, el poder duro, las economías, la anarquía, entre otras. La perspectiva analítica de Waltz permite comprender que los países en vía de desarrollo generan un poder "mediano" sobre otros Estados. Este es el caso de Brasil, que ha definido políticas de defensa nacional sobre la construcción de la soberanía, de los recursos naturales y del poder medio militar, características que le permiten tener un alcance regional y un liderazgo sobre esta región.

Brasil es el único país suramericano con una ley que obliga al Ministerio de Defensa a presentar directamente al Congreso una revisión del Libro blanco de defensa cada cuatro años. Esta ley establece explícitamente que la Estrategia Nacional de Defensa debe servir para planear anualmente el sector y brindar información detallada sobre estrategia, asuntos institucionales y material de las Fuerzas Armadas.

En Colombia, por su parte, no existe una orientación sobre las políticas de seguridad y defensa debido a la ausencia de procesos de consulta. Por lo tanto, se espera que el libro sea no solo un estímulo para que en el país se discutan temas de seguridad y defensa nacional, sino también que en este documento el gobierno pueda hacer una rendición de cuentas a la población en cuanto a la adecuación de la estructura de defensa, que es una política pública del Estado.

Por todo esto, la investigación sobre la creación de un libro blanco en Colombia es un tema muy especial, teniendo en cuenta que cada cuatro años el gobierno de turno debe elaborar un plan de desarrollo, donde consigna todas las políticas, intenciones e intereses nacionales para el periodo presidencial correspondiente. Dentro de este plan de desarrollo se define también un plan de seguridad y defensa, en el cual están consignados los objetivos, intenciones, metas, amenazas y todos aquellos aspectos relacionados con la defensa del país. Es decir, se trata de un documento muy similar a un libro blanco, si se compara con los conceptos que se presentan más adelante (Quintero \& Forero, 2018).

No obstante, a pesar de su importancia, en la búsqueda de literatura académica no se encontraron trabajos sobre la creación de un libro blanco en Colombia. Por esta razón, en este artículo se decidió simplificar la búsqueda y tomar por aparte los factores consignados en el libro blanco de Brasil (Álvarez \& Rodríguez, 2018). Pero, ¿por qué se analiza el caso de Brasil? Se estudia el Estado brasileño por el desarrollo de su industria militar y porque en la región es el Estado con mayor cantidad de recursos naturales, economía y sostenimiento público. Asimismo, el libro blanco brasileño permite identificar en un país suramericano los principales elementos constitutivos de la forma como un Estado define su identidad estratégica y de qué manera elige sus intereses de acuerdo con su contexto y problemas específicos.

Ahora bien, se debe señalar que ninguna política de defensa se puede formular sin saber previamente cuáles son las principales características y las amenazas del sistema internacional, la manera como está dividido el poder entre los actores y cómo afectan la cooperación 
dentro del ambiente estratégico. Precisamente sobre el contexto actual, Celso Amorío, quien ha sido ministro de Asuntos Exteriores de Brasil en dos oportunidades, subraya el impacto que tuvo la finalización de la Guerra Fría, cuando parecía que la unipolaridad traería estabilidad al sistema internacional. Sin embargo, agrega, después de los ataques del 11 de septiembre en Nueva York, Estados Unidos adoptó un nuevo concepto estratégico que trajo nuevamente inestabilidad al mundo (Quintero \& Forero, 2018).

En este tablero de operaciones, el libro blanco de defensa nacional brasileño aborda temas relacionados con el escenario estratégico para el siglo XXI: la estrategia y la política de defensa nacional; la modernización de las Fuerzas Armadas; los desarrollos tecnológicos del Estado; evalúa cuál es el respaldo económico del gasto en seguridad y defensa nacional; analiza cada una de las Fuerzas que componen el ente militar del Estado y las operaciones de paz y ayuda humanitaria. Asimismo, abarca sectores estratégicos para la defensa, remarcando los sectores nuclear, cibernético y espacial (Ripoll de Castro \& Quintero, 2018).

En este libro blanco de Brasil también se evidencian los cambios internos que ha tenido el Estado en relación con la protección del medioambiente en su política de Amazonia Azul y Amazonia Verde, una zona biodiversa y rica en recursos energéticos con reservas de petróleo. En otro frente estratégico, el documento define objetivos en áreas modernas como los estudios nucleares y de ciberseguridad, detalla las capacidades logísticas que tendrán las Fuerzas Armadas y precisa la ruta para que los países suramericanos establezcan alianzas para la producción de defensa. Además de definir una postura de cooperación para la paz y el desarrollo, se establecen los mecanismos necesarios para mantener una relación con los países vecinos y la comunidad internacional basada en el respeto mutuo y la confianza, lo cual evidencia que Brasil se enfoca en sus capacidades y no en responder a un enemigo (Quintero \& González, 2017).

Para ampliar su proyección, en el libro blanco el Estado brasileño promueve acciones que intensifiquen la participación en acciones humanitarias y misiones de paz. Al mismo tiempo, busca proyectar su poder participando en operaciones autorizadas por la Organización de las Naciones Unidas (ONU), ejecuta ejercicios militares con diferentes fuerzas armadas del mundo y mejora su participación en el grupo BRICS (Brasil, Rusia, India, China y Sur África), entre los cuales es uno de los más importantes participantes.

Estas características del libro blanco de Brasil se pueden enmarcar en la idea de que

solo existe soberanía efectivamente con una fuerte defensa, es decir, con Fuerzas Armadas adecuadamente equipadas y adiestradas, en condiciones de actuar de forma conjunta en cualquier escenario, en especial ante los escenarios de amenazas cada vez más difusas. (Ministerio de Defensa, Gobierno de Brasil, 2012. p. 55)

\section{Políticas de seguridad y defensa de Álvaro Uribe y Juan Manuel Santos}

Ahora bien, al comparar el libro blanco brasileño con las políticas que Colombia ha proyectado en los planes de desarrollo de los periodos presidenciales de Álvaro Uribe y Juan Manuel Santos, se encuentra que los dos protegen la seguridad y defensa del territorio nacional con todos sus problemas internos y externos. Por ejemplo, los dos planes tienen como objetivo general definir estrategias para enfrentar la difícil problemática de las drogas ilícitas, que ha sido determinante en el accionar de los grupos al margen de la ley. No obstante, cabe señalar que debido a la respuesta estratégica que el Estado planteó como contraofensiva a los grupos terro- 
ristas, los gobiernos han definido como objetivo general de estos planes fortalecer las garantías y procesos de la justicia en Colombia.

Asimismo, en esta comparación se encontró que uno de los objetivos del plan de seguridad del periodo del expresidente Uribe era desarrollar las zonas deprimidas y aquellas afectadas por el conflicto, aspecto que no se menciona en el plan del gobierno Santos. En contraste, el expresidente Juan Manuel Santos anexó a su plan de seguridad puntos relacionados con el proceso de paz, lo cual significaba una garantía al acuerdo que pondría fin a una guerra y al nacimiento de nuevas víctimas.

\section{La política de defensa brasileña}

Además de los aspectos que se mencionaron en el apartado anterior, Brasil creó el libro blanco con el ánimo de que el Parlamento y la sociedad brasileña en general discutieran el tema de defensa y contara con un mecanismo de rendición de cuentas a la sociedad sobre la adecuación de la estructura de defensa, que es uno de los objetivos establecidos por el poder público. En el libro se define la defensa nacional como las medidas y acciones que define el Estado en el campo militar con el propósito de preservar la soberanía y los intereses nacionales, en relación con los intereses de la región, como una zona de paz, resolución de conflictos de manera pacífica, respeto a los principios de la ONU y del derecho internacional. Además de este objetivo principal, consigna los siguientes objetivos secundarios:

- Defender los intereses nacionales y de las personas, así como los recursos brasileños en el exterior.

- Promover la estabilidad regional.

- Contribuir con el mantenimiento de la paz en la región.
- Profesionalizar las Fuerzas Armadas para así adecuarse de manera moderna y balanceada a los problemas del sistema internacional.

- Estructurar las Fuerzas Armadas para dotarlas de personal y planificación estratégica.

La Estrategia de Defensa Nacional esbozada en el libro blanco se basa en dos ejes fundamentales: el primero es emplear las Fuerzas Armadas para asegurar el cumplimiento de la constitución en tiempos de paz y guerra. El segundo es reorganizar la defensa del Estado brasileño para garantizar que las Fuerzas Armadas y el Estado funcionen como una república, de tal manera que la nación trabaje mano a mano con las clases sociales con el propósito de asegurar la integridad del territorio y la defensa de los intereses nacionales, las personas, los bienes y los recursos brasileños, así como garantizar la soberanía del país, aspectos que constituyen los marcos legales que orientan la organización y modernización en el ámbito militar brasileño (Torrijos \& Pérez, 2014).

Para ampliar su proyección internacional, por medio del libro blanco el Estado brasileño promueve operaciones que intensifican la participación en acciones humanitarias y misiones de paz. Asimismo, busca proyectar su poder participando en operaciones autorizadas por la ONU, ejecutando ejercicios militares con diferentes Fuerzas Armadas del mundo y mejorando su posición en el grupo BRICS, en el cual, como se mencionó, es uno de los miembros más importantes.

En este punto es necesario recordar que el objetivo general del Estado es proveer la seguridad y la defensa necesarias para que la sociedad pueda alcanzar sus objetivos. En este sentido, Brasil se cataloga en sus principios bási- 
cos como una república federativa que adopta el presidencialismo como sistema de gobierno ejecutivo legislativo y judicial, poderes que tienen su fuerza equilibrada. Esta federación, unida por estados, distrito federal y municipios, tiene como principios la soberanía, la ciudadanía, la dignidad de la persona humana, los valores sociales, la libre iniciativa y el pluralismo político. Asimismo, Brasil ejerce una completa y exclusiva soberanía y trabaja en pro de las acciones que fortalecen la cooperación y la confianza entre los Estados (Cujabante \& Quintero, 2019).

En concordancia con esta perspectiva, la política exterior brasileña considera el diálogo y la cooperación internacional instrumentos esenciales para superar los obstáculos y para generar confianza entre los Estados. Específicamente, la política de seguridad y defensa tiene once objetivos generales mediante los cuales Brasil espera lograr sus intereses a futuro, entre esos están: garantizar la soberanía, el patrimonio nacional y la integridad territorial; defender los intereses nacionales y las personas, los bienes y los recursos brasileños en el exterior, y contribuir con el mantenimiento de la paz y la seguridad internacional.

Otra de sus metas estratégicas en las políticas de seguridad y defensa es preparar a sus Fuerzas Armadas con capacidades adecuadas para garantizar la seguridad del país tanto en épocas de paz como en tiempos de crisis. De esa manera, también se proyecta como potencia, al menos regional, cuando dice contribuir a la paz y la seguridad internacional, interesante punto dentro de su política de seguridad como proyección de poder.

Finalmente, la tabla 1 sintetiza las características que Colombia tiene con respecto al libro

Tabla 1. Comparación entre las características del libro blanco de Brasil y la política de seguridad y defensa colombiana

\begin{tabular}{lc}
\hline Características del libro blanco de Brasil & Política de defensa y seguridad colombiana \\
\hline Defensa nacional & Sí \\
\hline Sistema internacional & Sí \\
\hline Ministerio de Defensa & Sí \\
\hline Estructura organizacional & Sí \\
\hline Consejo Militar de Defensa & No \\
\hline Estado Mayor Conjunto de las Fuerzas Armadas (EMCFA) & No \\
\hline Fuerzas Armadas (Ejército, Marina y Fuerza Aérea) & Sí \\
\hline Dirección de Operaciones Conjuntas (CHOC) & Sí \\
\hline Dirección de Asuntos Estratégicos (CAE) & Sí \\
\hline Dirección de Logística (CHLOG) & Sí \\
\hline Enseñanza en el ámbito de la defensa & Sí \\
\hline Sectores estratégicos a la defensa & Sí \\
\hline Sistemas de monitoreo y control & No \\
\hline Programas sociales de defensa & No \\
\hline Acciones subsidiarias y complementarias & No \\
\hline Relación de la defensa con los poderes constituidos & No \\
\hline Defensa y Derechos Humanos & Sí \\
\hline Defensa y desarrollo industrial & No \\
\hline Plan de Articulación y Equipamiento de Defensa (PAED) & No \\
\hline Base Industrial de Defensa (BID) & No \\
\hline Presupuesto de la defensa & Sí \\
\hline \multicolumn{1}{|l}{ Fuente: Elaborado por los autores con base en Ministerio de Defensa, Gobierno de Brasil. (2012). }
\end{tabular}


blanco de Brasil. Como se observa, si bien hay unos pocos aspectos que no se encuentran en la política de seguridad y defensa colombiana, esta sí tiene la mayoría de las características que consigna el libro blanco de Brasil.

\section{Conclusiones}

Con base en el análisis realizado en esta investigación se concluye que el tema de la seguridad y la defensa es muy importante en la agenda internacional. Por esta razón, los Estados lo toman en cuenta muy seriamente cuando definen sus relaciones diplomáticas en el sistema internacional, de allí que haya una constante ayuda, cooperación e interacción entre estos Estados. En este contexto, se concluye que Colombia tiene un déficit en sus políticas de seguridad y defensa, pues es uno de los Estados que no tienen un libro blanco de seguridad y defensa. Por esta razón, en este trabajo se plantean las siguientes conclusiones que pueden contribuir a su creación:

- Las definiciones que se encuentran sobre los libros blancos en el mundo son coherentes y concuerdan en temas como las amenazas internas y externas, así como en la elaboración de una estrategia de seguridad general.

- Los libros blancos de los Estados deben ser renovados periódicamente y conforme con el movimiento del sistema internacional en la región o en el mundo. Estas políticas tendrán que ser consecuentes con los intereses propios del Estado y con su búsqueda de la visión que tenga sobre el estado ideal.

- Los libros blancos deben ser un bien de la sociedad al alcance de todos los ciudadanos. Si bien es evidente que la política de seguridad interna debe tener el tratamiento de un asunto clasificado $y$, en consecuencia, no se puede publicar a la opinión general, el libro blanco debe ser público porque esto legitima las decisiones que se han acordado en el documento según los supuestos y los caminos establecidos.

- Los libros blancos deben tener una estructura física específica que es organizada y orientada por la Organización de los Estados Americanos (OEA).

- Colombia ha tenido varias políticas de seguridad y defensa referentes al conflicto armado de más de cincuenta años que ha enfrentado y que está llegando a su final. Sin embargo, esta política no está enmarcada en una política de seguridad general.

- El Estado colombiano no tiene una política de seguridad general que le permita tener una visión a futuro sobre la soberanía.

\section{Agradecimientos}

Los autores agradecen a la Escuela Militar de Cadetes “General José María Córdova” por el apoyo brindado para la realización de este artículo.

\section{Declaración de divulgación}

Los autores declaran que no existe ningún potencial conflicto de interés relacionado con el texto. Los puntos de vista y los resultados de este artículo pertenecen al autor y no reflejan necesariamente los de la Escuela Militar de Cadetes “General José María Córdova”.

\section{Financiamiento}

Los autores no declaran fuente de financiamiento para la realización de este artículo. 


\section{Sobre los autores}

Jorge Chacón Moreno es Alférez de la Escuela Militar de Cadetes, estudiante de la Facultad de Relaciones Internacionales, Escuela Militar de Cadetes "General José María Córdova”, Bogotá, D. C., Colombia.

Juan Rojas Azuero es Alférez de la Escuela Militar de Cadetes, estudiante de la Facultad de Relaciones Internacionales, Escuela Militar de Cadetes “General José María Córdova”, Bogotá, D. C., Colombia.

Sara Quintero Cordero es internacionalista. Magíster en Relaciones Internacionales de la Universidad de Buenos Aires. Docente e investigadora de la Facultad de Relaciones Internacionales de la Escuela Militar de Cadetes “General José María Córdova”. Investigadora principal del proyecto. Contacto: sara.quintero@esmic.edu.co

\section{Referencias}

Álvarez Calderón, C. E., \& Rodríguez Beltrán, C. A. (2018). Ecosistemas criminales: hábitats para la convergencia y la globalización desviada. Revista Científica General José María Córdova, 16(24), 1-30. https://doi.org/10.21830/19006586.352

Cujabante Villamil, X. A., \& Quintero Cordero, S. (2019). Desde los Estados Unidos y Rusia: una mirada a la R2P. Reflexión Política, 21(43), 9-21. https://doi.org/10.29375/01240781.3503

Grabendorff, W. (2010) Brasil: de coloso regional a potencia global. Revista Nueva Sociedad, 226, 188-171. Recuperado de https://nuso.org/media/ articles/downloads/3691_1.pdf

Ministerio de Defensa, Gobierno de Brasil. (2012). Libro blanco de Brasil [documento en línea].
Recuperado de https://www.defesa.gov.br/ arquivos/estado_e_defesa/livro_branco/lbdn_ esp_net.pdf

Quintero Cordero, S., \& Forero Garzón, M. (2018). Los libros blancos de defensa de Perú y Brasil: aspectos geopolíticos, seguridad regional e incidencia para Colombia. En A. Fernández Osorio \& C. Álvarez Calderón (Eds.), Pensamiento y cultura estratégica en Suramérica: un análisis de sus libros blancos de defensa (vol. 3. Hacia una gran estrategia en Colombia: construcción de política pública en seguridad y defensa, pp. 89-119). Bogotá, D. C.: Sello Editorial ESMIC. Recuperado de https://librosesmic.com/index. php/editorial/catalog/view/23/20/245-1

Quintero Cordero, S., \& González, M. (2017). La evolución de la seguridad en el Sistema Internacional Contemporáneo: un balance a partir de los casos de Estados Unidos y la Federación de Rusia. En Castillo Castañeda A. \& Niño González C. (Eds.), Nociones sobre seguridad y paz en las relaciones internacionales contemporáneas (pp. 447-472). Bogotá, D.C.: Ediciones USTA.

Ripoll de Castro, A., \& Quintero Cordero, S. (2018). Regionalismo latinoamericano: la Alianza del Pacífico y la ALADI: ¿Integraciones complementarias o estancamiento de la integración latinoamericana? En Pastrana Buelvas E. \& Ing Hans Blomeier (Eds.), La Alianza del Pacífico: ¿Atrapada en el péndulo del regionalismo y del interregionalismo? (pp. 223-243). Bogotá. D. C.: Opciones Gráficas.

Torrijos Rivera, V., \& Pérez Carvajal, A. (2014). Geopolítica sistémica aplicada: un modelo para entender las dinámicas cambiantes del sistema internacional. Revista Científica General José María Córdova, 12(14), 35-56. https://doi. org/10.21830/19006586.55

Waltz, K. N. (2000). Structural realism after the Cold War. International Security, 25(1) 5-41. 